Supporting Information

\title{
Elimination of Charge Recombination Centers in Metal Halide Perovskites by Strain
}

\author{
Lu Qiao $^{1}$, Wei-Hai Fang ${ }^{1}$, Run Long ${ }^{1 *}$, Oleg. V. Prezhdo ${ }^{2}$ \\ ${ }^{1}$ College of Chemistry, Key Laboratory of Theoretical \& Computational Photochemistry of Ministry \\ of Education, Beijing Normal University, Beijing, 100875, People's Republic of China \\ ${ }^{2}$ Department of Chemistry, University of Southern California, Los Angeles, California 90089, USA
}

\section{Simulation Methods}

In order to investigate the nonradiative charge trapping and recombination processes in the pristine $\mathrm{MAPbI}_{3}, \mathrm{I}_{\mathrm{i}}, \mathrm{I}_{\mathrm{i}} @ \mathrm{com}, \mathrm{MA}_{\mathrm{I}}$ and $\mathrm{MA}_{\mathrm{I}} @$ ten systems, the simualtions were performed by the combinaiton of nonadiabatic molecular (NA) dynamics (MD) ${ }^{1}$ and time-dependent (TD) density functional theory (DFT). ${ }^{2,3}$ NAMD is carried out using the quantum-classical decoherence-induced surface hopping (DISH) algorithm ${ }^{4}$ implemented with the Kohn-Sham (KS) forumation of TD-DFT. ${ }^{2,3}$

\section{Time-Domain Density Functional Theory}

In DFT, the electron density, $\rho(\mathbf{r}, \mathrm{t})$, is described by the sum of total densities of occupied singleelectron KS orbitals: ${ }^{5}$

$$
\rho(\mathbf{r}, t)=\sum_{i=1}^{N_{e}}\left|\varphi_{i}(\mathbf{r}, t)\right|^{2}
$$

Application of time-dependent variational principle to the KS orbital energy, we obtain a set of single-electron equations for the evolution of the KS orbitals: ${ }^{5,6}$

$$
i \hbar \frac{\partial \varphi_{i}(\mathbf{r}, t)}{\partial t}=H(\mathbf{r}, \mathbf{R}, t) \varphi_{i}(\mathbf{r}, t)
$$

\footnotetext{
*Corresponding author, E-mail: runlong@bnu.edu.cn
} 
Expansion of the time-dependent KS orbitals in the adiabatic KS orbital basis at a given nuclear configuration:

$$
\varphi_{i}(\mathbf{r}, t)=\sum_{i=1}^{N_{e}} c_{i}(t) \mid \tilde{\varphi}_{i}(\mathbf{r}, \mathbf{R}(t))
$$

Inserting eq S3 into eq S2 leads to equations for the expansion coefficients:

$$
i \hbar \frac{\partial}{\partial t} c_{j}(t)=\sum_{i} c_{i}(t)\left(\varepsilon_{i} \delta_{j i}+\mathbf{d}_{j i} \cdot \dot{\mathbf{R}}\right)
$$

Here, $\varepsilon_{i}$ is the energy of the adiabatic state $i$, and $\boldsymbol{d}_{j i} \cdot \dot{\boldsymbol{R}}$ is the NA coupling between state $i$ and $j$. The NA coupling arises because electronic wave functions depend on nuclear coordinates. The NA coupling is calculated numerically as the overlap of states $i$ and $j$ at sequential time steps: ${ }^{7}$

$$
\begin{aligned}
\mathbf{d}_{j i} \cdot \dot{\mathbf{R}} & =-i \hbar\left\langle\tilde{\varphi}_{j}(\mathbf{r}, \mathbf{R}(t))\left|\nabla_{R}\right| \tilde{\varphi}_{i}(\mathbf{r}, \mathbf{R}(t))\right\rangle \cdot \frac{\mathrm{d} \mathbf{R}}{\mathrm{dt}} \\
& =-i \hbar\left\langle\tilde{\varphi}_{j}(\mathbf{r}, \mathbf{R}(t))\left|\frac{\partial}{\partial t}\right| \tilde{\varphi}_{i}(\mathbf{r}, \mathbf{R}(t))\right\rangle \\
& \approx-\frac{i \hbar}{2 \Delta t}\left(\left\langle\tilde{\varphi}_{j}(t) \mid \tilde{\varphi}_{i}(t+\Delta t)\right\rangle-\left\langle\tilde{\varphi}_{j}(t+\Delta t) \mid \tilde{\varphi}_{i}(t)\right\rangle\right)
\end{aligned}
$$

\section{NAMD by Decoherence-Induced Surface Hopping}

Surface hopping is a method to describe the state-specific back-reaction of electrons onto the nuclei, which can reflect trajectory branching. When bath-induced coherence loss is slower than transitions between states, standard quantum-classical models are appropriate. However, when the loss of coherence becomes faster than the transitions, decoherence should be considered explicitly. $\mathrm{DISH}^{4}$ offers a conceptually sound and efficient methodology to introduce decoherence effects into quantum-classical nonadiabatic molecular dynamics simulations. Decoherence in the quantum subsystem is modeled as collapse of the wavefunction onto basis states, which are typically taken to 
be stationary adiabatic states. The stochastic collapse gives rise to trajectory branching and a surface hopping algorithm..

Decoherence is described as pure-dephasing according to the optical theory, ${ }^{8}$ which can be quantized by double integration of energy gap of unnormalized fluctuations autocorrelation function $(\mathrm{ACF}), C_{u n}(t)$. The ACF represents the phonon-induced fluctuation of the energy gap between two states, $\Delta E(t)$. The normalized, $C(t)$, and unnormalized, $C_{u n}(t)$, ACFs as well as the pure-dephasing funcitons are defined by eqs S6-S8:

$$
\begin{gathered}
C(t)=\frac{\langle\Delta E(t) E \Delta E(0)\rangle_{T}}{\left\langle\Delta E^{2}(0)\right\rangle_{T}}=\frac{C_{u n}(t)}{\left\langle\Delta E^{2}(0)\right\rangle_{T}} \\
D_{\text {сити }}(t)=\exp [-g(t)] \\
g(t)=\int_{0}^{t} d \tau_{1} \int_{0}^{\tau_{1}} d \tau_{2} C_{u n}\left(\tau_{2}\right)
\end{gathered}
$$

In geenral, fitting Eq. (8) to a Gaussian gives the pure-dephasing (decoherence) time.

Fourier transform of the ACF leads to the spectral density:

$$
I(\omega)=\left|\frac{1}{\sqrt{2 \pi}} \int_{-\infty}^{\infty} d t e^{-i \omega t} C(t)\right|^{2}
$$

Spectral density characterizes the phonon modes participating in the electronic transition between the electronic states. 

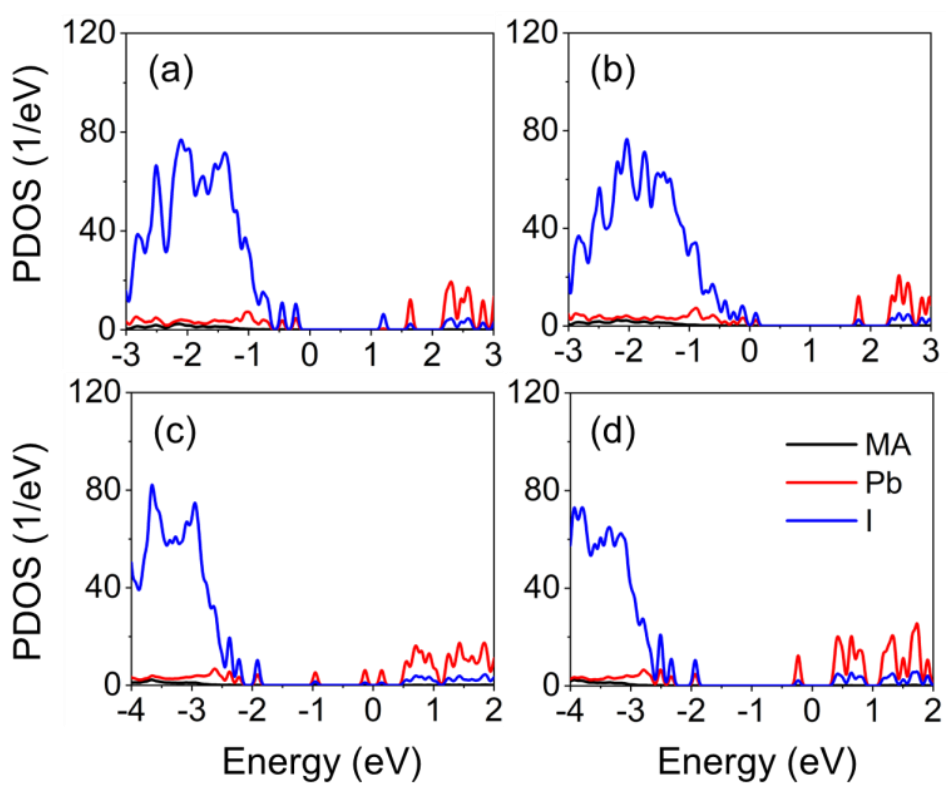

Figure S1. Projected densities of states (PDOS) of (a) $\mathrm{I}_{\mathrm{i}}$, (b) $\mathrm{I}_{\mathrm{i}} @$ com, (c) $\mathrm{MA}_{\mathrm{I}}$, and (d) $\mathrm{MA}_{\mathrm{I}} @$ ten systems calculated using the HSE06+SOC functional at the $\Gamma$-point. The obtained results are consistent with the data calculated using the PBE functional. The trap states created by te $\mathrm{I}_{\mathrm{i}}$ and $M \mathrm{~A}_{\mathrm{I}}$ defects are eliminiated under the compressive and tensile strain, respectively.

(a)

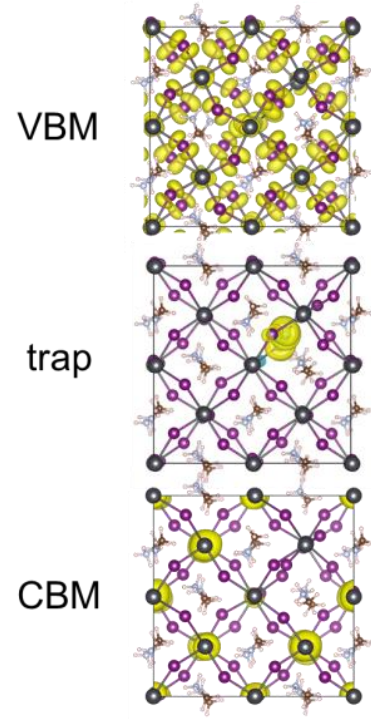

(b)

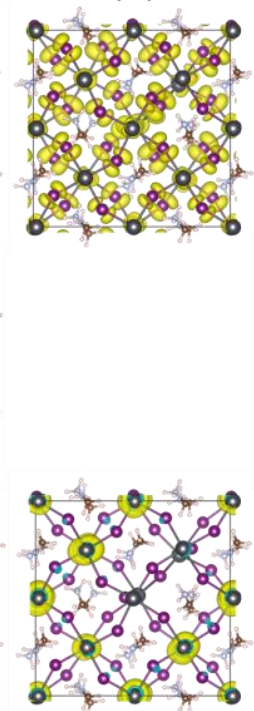

(c)

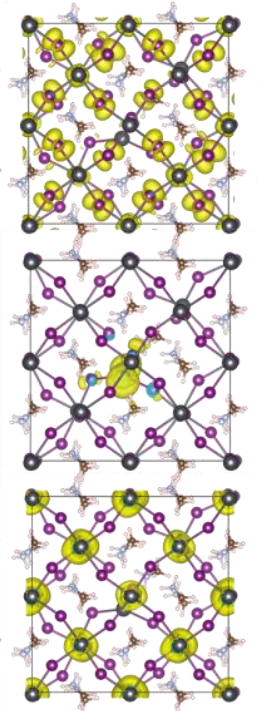

(d)

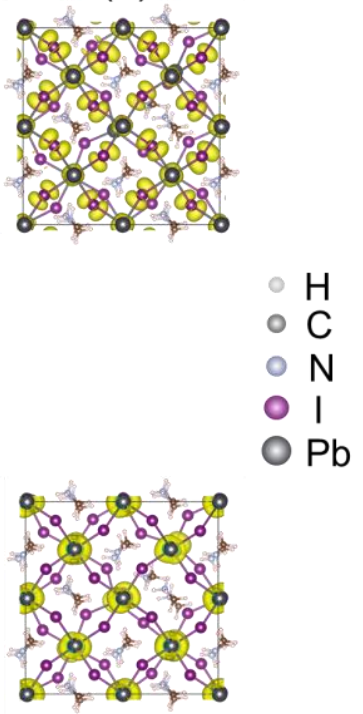

Figure S2. Charge density of the key states in (a) $\mathrm{I}_{\mathrm{i}}$, (b) $\mathrm{I}_{\mathrm{i}} @$ com, (c) $M \mathrm{~A}_{\mathrm{I}}$ and (d) $\mathrm{MA} \mathrm{A}_{\mathrm{I}} @$ ten systems, calculated at the HSE06+SOC level. 
(a) $\mathrm{Br}_{\mathrm{i}}$

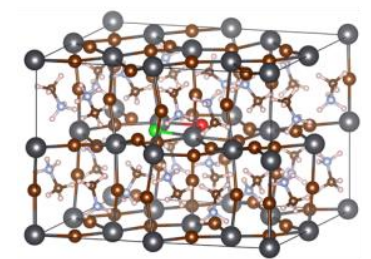

(c) $\mathrm{MA}_{\mathrm{Br}}$

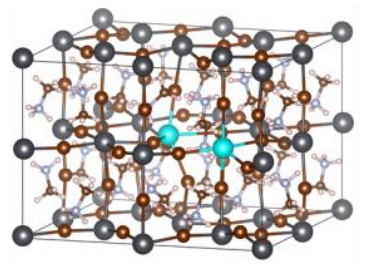

0 ○ 0

$\mathrm{HCN} \mathrm{BrPb}$ (b) $\mathrm{Br}_{i} @ \mathrm{com}$

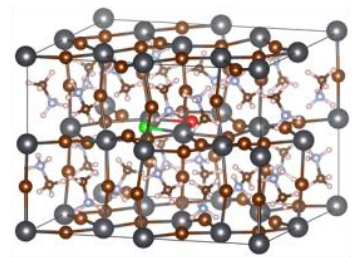

(d) $\mathrm{MA}_{\mathrm{Br}} @$ ten

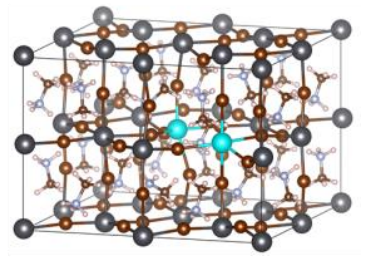

Figure S3. Optimized structures of (a) $\mathrm{MAPbBr}_{3}$ containing a bromine interstitial defect colored in green $\left(\mathrm{Br}_{\mathrm{i}}\right)$, (b) $\mathrm{MAPbBr} 3$ with $\mathrm{Br}_{\mathrm{i}}$ under $2 \%$ compressive strain $\left(\mathrm{Br}_{\mathrm{i}} @\right.$ com), (c) $\mathrm{MAPbBr}$ with a bromine atom replaced by a $\mathrm{MA}$ cation $\left(\mathrm{MA}_{\mathrm{Br}}\right)$, (d) $\mathrm{MAPbBr}_{3}$ containing $\mathrm{MA}_{\mathrm{Br}}$ under $2 \%$ tensile strain (MABr@ten). The Br interstitial is $3.452 \AA$ from an adjacent bromine atom in red. After compression, the $\mathrm{Br}-\mathrm{Br}$ distance shortens to $3.383 \AA$ in $\mathrm{Br}_{\mathrm{i}} @$ com. The two $\mathrm{Pb}$ atoms colored in blue around $\mathrm{MA}_{\mathrm{Br}}$ move away from each other after application of the tensile strain. This $\mathrm{Pb}-\mathrm{Pb}$ distance increases from $6.927 \AA$ in $\mathrm{MA}_{\mathrm{Br}}$ to $7.051 \AA$ in $\mathrm{MA} \mathrm{Br}_{\mathrm{Br}} @$ ten. 

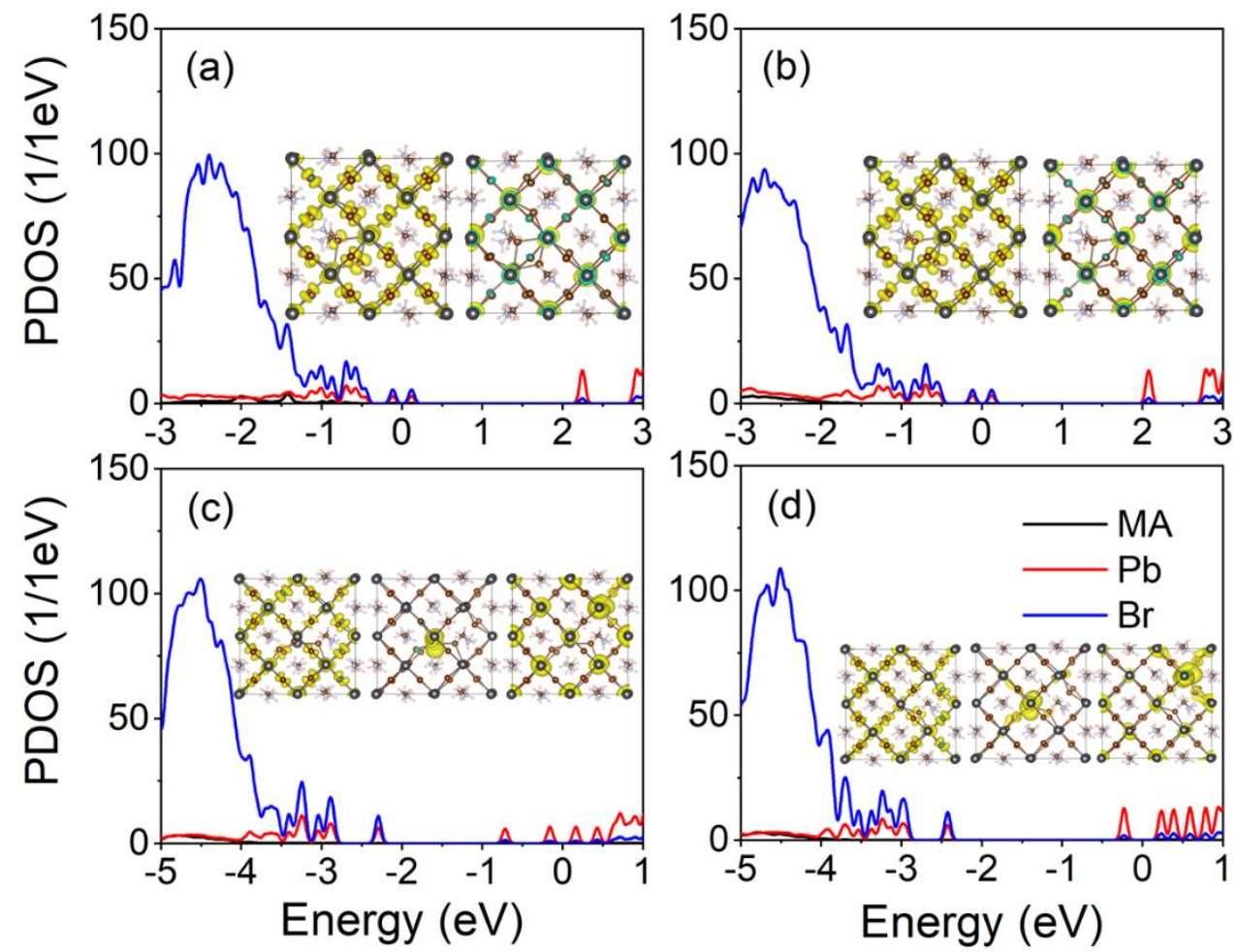

Figure S4. Projected densities of states (PDOSs) and charge densities of (a) $\mathrm{Br}_{\mathrm{i}},(\mathbf{b}) \mathrm{Br}_{\mathrm{i}} @ \mathrm{com}$, (c) $\mathrm{MA}_{\mathrm{Br}}$ and (d) $\mathrm{MA}_{\mathrm{Br}} @$ ten systems calculated by the HSE06+SOC method at the direct bandgap. Electrons at the CBM are supported by $\mathrm{Pb}$ atoms, while holes at the VBM are located on $\mathrm{Br}$ atoms. The $\mathrm{Br}_{\mathrm{i}}$ defect exhibits no trap states within the bandgap either with or without the $2 \%$ compressive strain, because the $\mathrm{Br}-\mathrm{Br}$ distance is too large to form a halogen dimer that is the source of the trap state in the $\mathrm{I}_{\mathrm{i}}$ system. The $\mathrm{MA}_{\mathrm{Br}}$ defect generates a hole trap state near the CBM. Under the $2 \%$ tensile strain the trap state merges with the $\mathrm{CBM}$, similarly to $\mathrm{MAPbI}_{3}$. The charge densities of the trap state concentrate on the $\mathrm{Pb}$ atoms around the $\mathrm{MA}_{\mathrm{Br}}$ defect in the $\mathrm{MA}_{\mathrm{Br}}$ and $\mathrm{MA} \mathrm{A}_{\mathrm{Br}} @$ ten systems. 


\section{Kinetics Models}

The schematics and basis sets for the charge recombination processes in the pristine $\mathrm{MAPbI}_{3}$, $\mathrm{I}_{\mathrm{i}} @$ com and MA $\mathrm{I} @$ ten systems are depicted in Figure S5. The corresponding information for the $\mathrm{I}_{\mathrm{i}}$ and $\mathrm{MA}_{I}$ systems that contain midgap states is shown in Figures S6 and S7, respectively. The ground, excited s and trap states are denoted as [GS], [ES] and [trap], respectively.

The evolution of populations of the excited and trap states during the charge trapping and recombination processes are shown in Figures S8-S10. Fitting the data to the exponential function or its short-time approximation, $P(t)=\exp (-k t) \approx 1-k t$, gives the transition rates $k_{(\mathrm{cbm} \rightarrow \mathrm{vbm})}$, $k_{(\mathrm{cbm} \rightarrow \operatorname{trap})}$ and $k_{(\mathrm{vbm} \rightarrow \operatorname{trap})}$ between pairs of electronic states, as shown in Figures S8-S10. VBM means the valence band maximum, while CBM means the conduction band minimum. 


\section{Pristine MAPbI $3, I_{i} @$ com and MAI @ten systems}

Electron-hole recombination takes place between the CBM and VBM, since there are no midgap trap states, Figure $2 b$, d of the main text. The corresponding kinetic equations S10 and S11, and their solution S11 and S12, give evolution of the key states shown in Figure 6b, d. Figure S8 displays the NAMD data used to obtain the state-to-state rate constants.

\section{Coupled kinetic equations:}

$$
\begin{aligned}
& \frac{\mathrm{d}[\mathrm{ES}]}{\mathrm{dt}}=-\mathrm{k}_{(\mathrm{cbm} \rightarrow \mathrm{vbm})}[\mathrm{ES}] \\
& \frac{\mathrm{d}[\mathrm{GS}]}{\mathrm{dt}}=\mathrm{k}_{(\mathrm{cbm} \rightarrow \mathrm{vbm})}[\mathrm{ES}]
\end{aligned}
$$

Solution of above equations:

$$
\begin{aligned}
& {[E S]=\mathrm{e}^{\left.-\mathrm{k}_{(\mathrm{cbm} \rightarrow \mathrm{vbm})}\right)^{* \mathrm{t}}}} \\
& {[\mathrm{GS}]=1-\mathrm{e}^{-\mathrm{k}_{(\mathrm{cbm} \rightarrow \mathrm{vbm})} * \mathrm{t}}} \\
& \text { Ground State Excited State } \\
& \text { CBM } 2 \\
& \mathrm{GS}=[1,-1]
\end{aligned}
$$
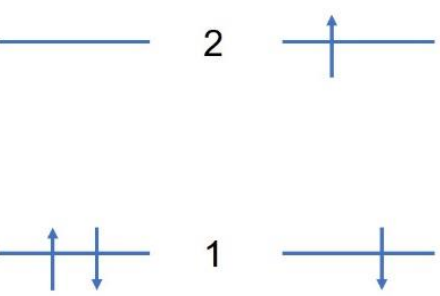

Figure S5. Electronic states involved in the electron-hole recombination across the CBM-VBM energy gap in the pristine $\mathrm{MAPbI}_{3}, \mathrm{I}_{\mathrm{i}} @$ com, and MAI@ ten systems. 


\section{Ii System}

The $I_{i}$ defect creates a singly occupied midgap state that can serve as both electron and hole trap, Figure 2a. Since the trap state is much closer than to the VBM than CBM, hole trapping proceeds much faster than electron trapping, as analyzed in our previous work, ${ }^{1}$ and therefore, we focus on the dominant hole-trap-mediated charge recombination mechanism. The kinetics describing the electronhole recombination in the $I_{i}$ system are given by equations S14-S16. The solution is presented in equations S17-S19 and is plotted in Figure 6a of the main text. Figure S6 displays the basis set. Figure S9 shows the NAMD data used to obtain the transition rate constants.

\section{Coupled kinetic equations:}

$$
\begin{aligned}
& \frac{\mathrm{d}[\mathrm{ES}]}{\mathrm{dt}}=-\left(\mathrm{k}_{(\mathrm{cbm} \rightarrow \mathrm{vbm})}+\mathrm{k}_{(\mathrm{vbm} \rightarrow \operatorname{trap})}\right)[\mathrm{ES}] \\
& \frac{\mathrm{d}[\mathrm{trap}]}{\mathrm{dt}}=\mathrm{k}_{(\mathrm{vbm} \rightarrow \operatorname{trap})}[\mathrm{ES}]-\mathrm{k}_{(\mathrm{cbm} \rightarrow \operatorname{trap})}[\mathrm{trap}] \\
& \frac{\mathrm{d}[\mathrm{GS}]}{\mathrm{dt}}=\mathrm{k}_{(\mathrm{cbm} \rightarrow \mathrm{vbm})}[\mathrm{ES}]+\mathrm{k}_{(\mathrm{cbm} \rightarrow \operatorname{trap})}[\mathrm{trap}]
\end{aligned}
$$

\section{Solution of above equations:}

$$
\begin{aligned}
& {[E S]=\mathrm{e}^{-\left(\mathrm{k}_{(\mathrm{cbm} \rightarrow \mathrm{vbm})}+\mathrm{k}_{(\mathrm{vbm} \rightarrow \operatorname{trap})}\right) * \mathrm{t}}} \\
& {[\text { trap }]=\frac{\mathrm{k}_{(\mathrm{vbm} \rightarrow \operatorname{trap})}}{\left(\mathrm{k}_{(\mathrm{cbm} \rightarrow \mathrm{vbm})}-\mathrm{k}_{(\mathrm{cbm} \rightarrow \text { trap })}+\mathrm{k}_{(\mathrm{vmb} \rightarrow \operatorname{trap})}\right)}\left(e^{-k_{(\text {cbm } \rightarrow \text { trap })^{* t}}}-\right.} \\
& \left.e^{-\left(k_{(c b m \rightarrow v b m)}+k_{(v b m \rightarrow t r a p)}\right) * t}\right) \\
& {[\mathrm{GS}]=1-\frac{\mathrm{k}_{(\mathrm{vbm} \rightarrow \operatorname{trap})}}{\left(\mathrm{k}_{(\mathrm{cbm} \rightarrow \mathrm{vbm})}-\mathrm{k}_{(\mathrm{cbm} \rightarrow \operatorname{trap})}+\mathrm{k}_{(\mathrm{vbm} \rightarrow \operatorname{trap})}\right)}\left(e^{-k_{(\text {cbm } \rightarrow \text { trap })^{* t}}}\right)-} \\
& \frac{\left(\left(k_{(c b m \rightarrow v b m)}-k_{(c b m \rightarrow t r a p)}\right.\right.}{\left(k_{(c b m \rightarrow v b m)}-k_{(c b m \rightarrow t r a p)}+k_{(v b m \rightarrow \text { trap })}\right)}\left(e^{-\left(k_{(c b m \rightarrow v b m)}+k_{(v b m \rightarrow \text { trap })}\right) * t}\right)
\end{aligned}
$$

\section{Ground State Hole Trap Excited State}

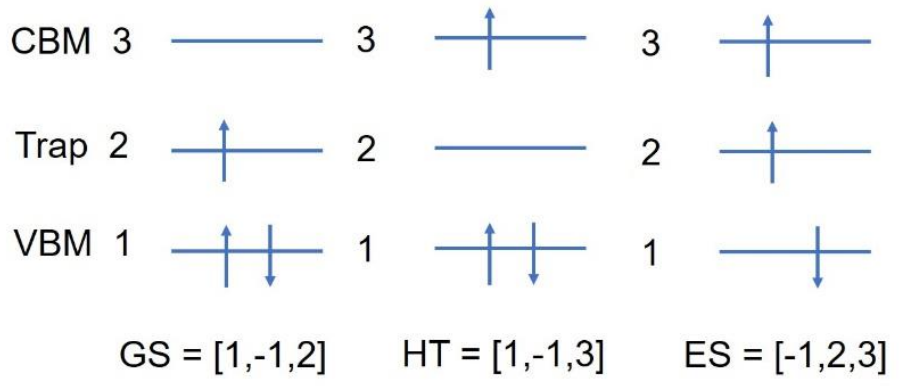

Figure S6. Electronic states involved in the hole-trap-assisted charge recombination in the $\mathrm{I}_{\mathrm{i}}$ system. 


\section{MAI system}

The $\mathrm{MA}_{\mathrm{I}}$ defect creates a doubly occupied midgap state, Figure $2 \mathrm{c}$ of the main manuscript. Even though the state is closer to the CBM than the VBM, it can only act as a hole trap since it is already fully occupied. The kinetics describing the electron-hole recombination in the $\mathrm{MA}_{\mathrm{I}}$ system are given by equations S20-S22. The solution is presented in equations S23-S25 and is plotted in Figure 6c of the main text. Figure S7 displays the basis set. Figure S10 shows the NAMD data used to obtain the transition rate constants.

\section{Coupled kinetic equations:}

$$
\begin{aligned}
& \frac{\mathrm{d}[\mathrm{ES}]}{\mathrm{dt}}=-\left(\mathrm{k}_{(\mathrm{cbm} \rightarrow \mathrm{vbm})}+\mathrm{k}_{(\mathrm{vbm} \rightarrow \operatorname{trap})}\right)[\mathrm{ES}] \\
& \frac{\mathrm{d}[\mathrm{trap}]}{\mathrm{dt}}=\mathrm{k}_{(\mathrm{vbm} \rightarrow \operatorname{trap})}[\mathrm{ES}]-\mathrm{k}_{(\mathrm{cbm} \rightarrow \operatorname{trap})}[\mathrm{trap}] \\
& \frac{\mathrm{d}[\mathrm{GS}]}{\mathrm{dt}}=\mathrm{k}_{(\mathrm{cbm} \rightarrow \mathrm{vbm})}[\mathrm{ES}]+\mathrm{k}_{(\mathrm{cbm} \rightarrow \operatorname{trap})}[\mathrm{trap}]
\end{aligned}
$$

\section{Solution of above equations:}

$$
\begin{aligned}
& {[E S]=\mathrm{e}^{-\left(\mathrm{k}_{(\mathrm{cbm} \rightarrow \mathrm{vbm})}+\mathrm{k}_{(\mathrm{vbm} \rightarrow \mathrm{trap})}\right) * \mathrm{t}}} \\
& {[\text { trap }]=\frac{\mathrm{k}_{(\mathrm{vbm} \rightarrow \operatorname{trap})}}{\left(\mathrm{k}_{(\mathrm{cbm} \rightarrow \mathrm{vbm})}-\mathrm{k}_{(\mathrm{cbm} \rightarrow \operatorname{trap})}+\mathrm{k}_{(\mathrm{vmb} \rightarrow \operatorname{trap})}\right)}\left(e^{-k_{(\mathrm{cbm} \rightarrow \text { trap })^{* t}}}-\right.} \\
& \left.e^{-\left(k_{(c b m \rightarrow v b m)}+k_{(v b m \rightarrow t r a p)}\right) * t}\right) \\
& {[\mathrm{GS}]=1-\frac{\mathrm{k}_{(\mathrm{vbm} \rightarrow \operatorname{trap})}}{{\left(\mathrm{k}_{(\mathrm{cbm} \rightarrow \mathrm{vbm})} \mathrm{k}_{(\mathrm{cbm} \rightarrow \operatorname{trap})}+\mathrm{k}_{(\mathrm{vbm} \rightarrow \operatorname{trap})}\right)}}\left(e^{-k_{(\text {cbm } \rightarrow \text { trap })^{* t}}}\right)-} \\
& \frac{\left(\left(k_{(c b m \rightarrow v b m)}-k_{(c b m \rightarrow t r a p)}\right.\right.}{\left(k_{(c b m \rightarrow v b m)}-k_{(c b m \rightarrow t r a p)}+k_{(v b m \rightarrow \text { trap })}\right)}\left(e^{-\left(k_{(c b m \rightarrow v b m)} k_{(v b m \rightarrow \text { trap })}\right) * t}\right) \\
& \text { Ground State Hole Trap Excited State } \\
& \text { CBM } 3 \\
& \uparrow \quad \uparrow \\
& \text { Trap } 2 \uparrow \downarrow \\
& \text { VBM } 1
\end{aligned}
$$
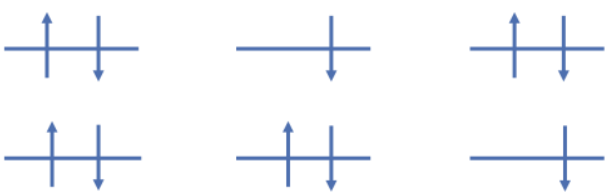

Figure S7. Electronic states involved in the hole-trap-assisted charge recombination in the $\mathrm{MA}_{\mathrm{I}}$ system. 


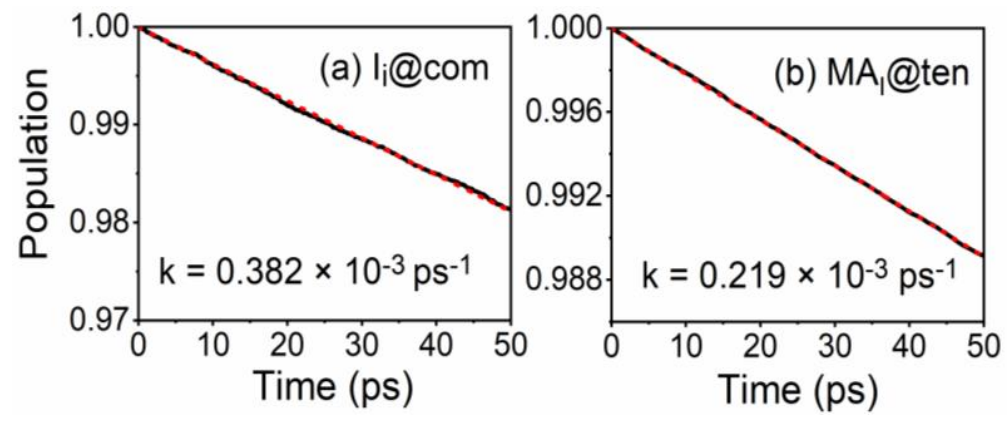

Figure S8. The population decay of the excited state during the transition from the CBM to VBM in (a) $\mathrm{I}_{\mathrm{i}} @$ com and (b) MA $\mathrm{M} @$ ten systems.

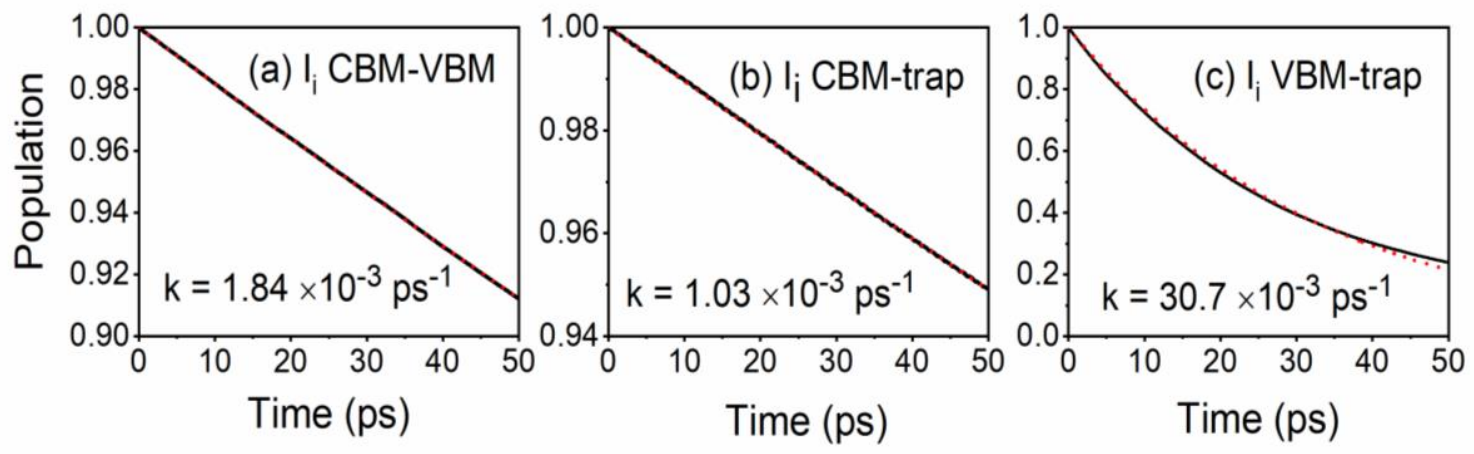

Figure S9. The populations of the key states in the $I_{i}$ system. (a) The population decay of the excited state due to transition from the CBM to the VBM. (b) The CBM population decay due to transition from the CBM to the trap state. (c) The trap state population decay due to transition from the trap state to the VBM.
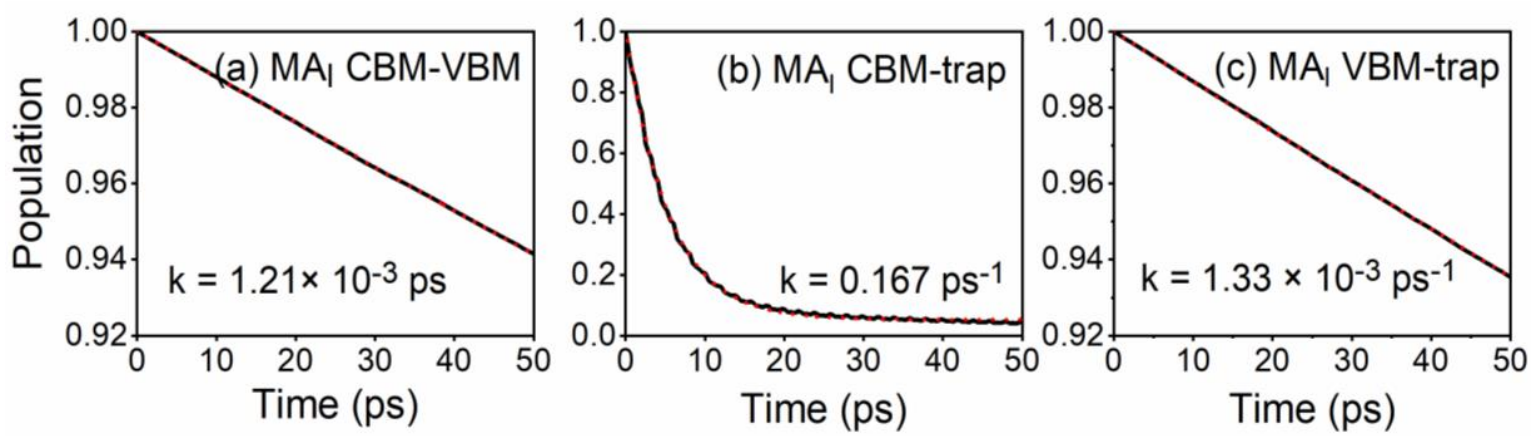

Figure S10. The populations of the key states in the MAI system. (a) The population decay of the excited state due to transition from the CBM to VBM. (b) The CBM population decay due to transition from the CBM to the trap state. (c) The trap state population decay due to transition from the trap state to the VBM. 


\section{References:}

(1) He, J.; Fang, W.-H.; Long, R. Unravelling the Effects of Oxidation State of Interstitial Iodine and Oxygen Passivation on Charge Trapping and Recombination in $\mathrm{CH}_{3} \mathrm{NH}_{3} \mathrm{PbI}_{3}$ Perovskite: a TimeDomain Ab Initio Study. Chem. Sci. 2019, 10, 10079-10088.

(1) Jasper, A. W.; Nangia, S.; Zhu, C. Y.; Truhlar, D. G. Non-Born-Oppenheimer Molecular Dynamics. Acc. Chem. Res. 2006, 39, 101-108.

(2) Craig, C. F.; Duncan, W. R.; Prezhdo, O. V. Trajectory Surface Hopping in the Time-Dependent Kohn-Sham Approach for Electron-Nuclear Dynamics. Phys. Rev. Lett. 2005, 95, 163001.

(3) Fischer, S. A.; Habenicht, B. F.; Madrid, A. B.; Duncan, W. R.; Prezhdo, O. V. Regarding the Validity of the Time-Dependent Kohn-Sham Approach for Electron-Nuclear Dynamics Via Trajectory Surface Hopping. J. Chem. Phys. 2011, 134, 024102.

(4) Jaeger, H. M.; Fischer, S.; Prezhdo, O. V. Decoherence-Induced Surface Hopping. J. Chem. Phys. 2012, 137, 22A545.

(5) Marques, M. A. L.; Gross, E. K. U. Time-Dependent Density Functional Theory. Annu. Rev. Phys. Chem. 2004, 55, 427-455.

(6) Baer, R.; Neuhauser, D. Real-Time Linear Response for Time-Dependent Density-Functional Theory. J. Chem. Phys. 2004, 121, 9803-9807.

(7) Hammes - Schiffer, S.; Tully, J. C. Proton transfer in solution: Molecular dynamics with quantum transitions. The Journal of Chemical Physics 1994, 101, 4657-4667.

(8) Mukamel, S. Principles of Nonlinear Optical Spectroscopy; Oxford University Press: New York. 1995. 